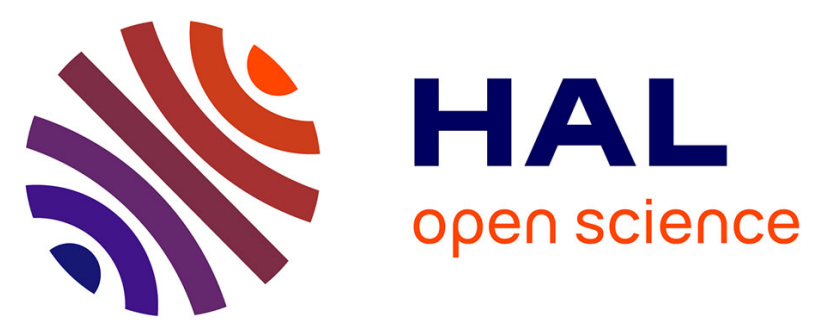

\title{
Inverse Identification at Very High Strain Rate of the Johnson-Cook Constitutive Model on the Ti-6Al-4V Alloy With a Specially Designed Direct-impact Kolsky Bar Device
}

\author{
Xiaoli Guo, Thomas Heuzé, Ramzi Othman, Guillaume Racineux
}

\section{To cite this version:}

Xiaoli Guo, Thomas Heuzé, Ramzi Othman, Guillaume Racineux. Inverse Identification at Very High Strain Rate of the Johnson-Cook Constitutive Model on the Ti-6Al-4V Alloy With a Specially Designed Direct-impact Kolsky Bar Device. Strain, 2014, 50 (6), pp.527-538. 10.1111/str.12114 . hal-01155471

\author{
HAL Id: hal-01155471 \\ https://hal.science/hal-01155471
}

Submitted on 9 Sep 2019

HAL is a multi-disciplinary open access archive for the deposit and dissemination of scientific research documents, whether they are published or not. The documents may come from teaching and research institutions in France or abroad, or from public or private research centers.
L'archive ouverte pluridisciplinaire HAL, est destinée au dépôt et à la diffusion de documents scientifiques de niveau recherche, publiés ou non, émanant des établissements d'enseignement et de recherche français ou étrangers, des laboratoires publics ou privés. 


\title{
Inverse Identification at Very High Strain Rate of the Johnson-Cook Constitutive Model on the Ti-6Al-4V Alloy With a Specially Designed Direct-impact Kolsky Bar Device
}

\author{
X. Guo*, T. Heuzé*, R. Othman ${ }^{\dagger}$ and G. Racineux* \\ *Research Institute in Civil and Mechanical Engineering (GeM, UMR 6 I 83 CNRS), École Centrale de Nantes, I rue de la Noë, 4432 I, Nantes cedex \\ 3, France \\ †Mechanical engineering department, Faculty Engineering, King Abdulaziz University, P.O.BOX, 80248, Jeddah, 2I589, Saudi Arabia
}

\begin{abstract}
In the present work, an inverse identification of the Johnson-Cook constitutive model is performed on the titanium alloy Ti$6 \mathrm{Al}-4 \mathrm{~V}$ at three strain rates until about $2 \cdot 10^{4} \mathrm{~s}^{-1}$ (till about $1.1 \cdot 10^{4} \mathrm{~s}^{-1}$ of plastic strain rate) on a specially designed direct impact Kolsky bar device. First, the design of such a device must meet several criteria, and is shown to be the solution of an optimization problem. A systematic design procedure for such a device is then introduced. Second, an inverse analysis using the finite element code ABAQUS is carried out to identify the Johnson-Cook parameters on experimental data obtained with the designed system.
\end{abstract}

KEY WORDS: direct impact Kolsky bar, high strain rate, inverse analysis, Johnson-Cook constitutive model

\section{Introduction}

The study of the material performance at high strain rate is an important topic in material sciences, which finds applications in different areas such as the defence, transports and material processing. In material forming processes, machining operations or impacting, the material can undergo high strain rates [1]. For instance in Electromagnetic Material Forming, in Shot Peening or Laser Shock Peening, the strain rates can range from $10^{4}$ to $10^{6} \mathrm{~s}^{-1}$ [2-7]. Compared to quasi-static situations, the material exhibits some viscous effects at high strain rate. But in dynamic processes, the range of achievable strain rate can be very large. Therefore, finding a constitutive model, calibrated on experimental data and being predictive for a wide range of strain rate remains a challenge.

Experimental data defined on such a wide range are needed on the one hand to better understand and characterize the behaviour of the material and on the other hand to calibrate constitutive models required to perform numerical simulations. Different experimental methods and equipments can be chosen according to the expected level of strain rate. Tests at strain rate up to $50 \mathrm{~s}^{-1}$ can be performed with a machine using mechanic or hydraulic loading [8, 9]. A conventional tensometer can measure the properties in tension, compression and shear [1]. A modified servo-hydraulic machine enables to attain a strain rate of approximately $10^{2} \mathrm{~s}^{-1}$ [10]. A bar instrumented with two gauges is mounted between the punch and the specimen to measure the force, while the strain in the specimen is captured via a high-speed camera [10]. As the rate increases, the wave propagation should be considered. Thus, devices like the classical Hopkinson bar can be adopted to perform the tests. The split
Hopkinson pressure bar (SHPB), also known as the Kolsky bar $[1,11]$, consists mainly of a projectile, the incident and transmitted bars, and can perform tests in compression [12-14], tension [15], torsion [16] or bending [1, 8]. Moreover, with some modifications on the conventional SHPB, it has been shown that the achievable strain rate can be largely extended $[1,17,18]$. One modification is to remove the incident bar so that the projectile impacts the specimen directly [8]. Therefore, the restrictions on the strain rate raised by the consistency between the impact velocity of the projectile and the strength of the incident bar disappear. With this direct-impact configuration, Gorham [1] has performed tests at a strain rate of $4 \cdot 10^{4} \mathrm{~s}^{-1}$ on tungsten alloy, titanium alloy (Ti-6Al-4 V) and pure copper. The other modification that can be made to increase the strain rate is to largely decrease the dimensions of the specimen and thus these of the SHPB device, usually referred to as the miniaturized SHPB [8]. Kamler [18] has developed a miniaturized system to perform experiments on copper and has reached strain rates ranging from $6 \cdot 10^{3}$ to $4 \cdot 10^{5} \mathrm{~s}^{-1}$. Casem et al. [17] have achieved strain rate higher than $10^{5} \mathrm{~s}^{-1}$ on the 6061-T6 aluminium alloy, with a very small bar of $1.6 \mathrm{~mm}$ in diameter.

Obtaining the stress-strain curve of the specimen material with the Hopkinson bar test is usually based on two assumptions $[8,11,14]$ : the unidimensional wave propagation throughout the device and the achievement of the force equilibrium within the specimen. The first assumption implies that the lateral effects in the device should be insignificant compared to longitudinal ones. The second assumption requires a very short duration to achieve the force equilibrium within the specimen with respect to the characteristic time, associated to a wave round trip within the projectile. In the design of a SHPB device, some criteria 
and empirical limits are usually defined to set the dimensions of the components so as to ensure these assumptions $[8,19$, 20]. But all the required criteria are not always cited or used; moreover, no practical or straightforward flowchart is available to help in the design. A systematic procedure needs to be strictly followed for the design in order to satisfy the requirements and to ensure the accuracy of the measurements and calculations.

As the strain rate increases, these two assumptions become more difficult to enforce; in particular, the second one has to be replaced by the writing of the motion equations. Therefore, we are led to perform an inverse analysis involving a numerical dynamic analysis of the whole device with a constitutive model for the specimen to describe its mechanical behaviour. Several constitutive equations are available to describe the rate dependence of the material. Actually, at least three models are mostly adopted for metallic materials. The Cowper-Symonds model [21] proposes to account for the rate dependence by scaling the yield stress with a strain rate-dependent factor to define a dynamic one; Zerilli and Armstrong [22] propose an expression of the flow stress based on simplified dislocation mechanics coupling the strain hardening, rate dependence and thermal effects; the Johnson-Cook model [23] consists of an empirical expression of the flow stress including as well the strain hardening, rate dependence and thermal effects, but with fewer parameters, making it a popular model. The parameters of this latter model identified for Ti-6Al-4 V are usually based on the stress-strain relations obtained at strain rates ranging from 1 to $10^{3} \mathrm{~s}^{-1}$ [24-26]. Although experimental data recorded at high strain rates are available in the literature on this alloy [1], the Johnson-Cook model has not been calibrated on them so far; only parameters identified at low and moderate strain rates are usually used in numerical applications [24-26].

The aim of this paper is threefold. First, a systematic procedure is derived to design a direct impact device in order to meet a set of criteria. The designing criteria are introduced in the second section; the design of such a device is shown to be the solution of an optimization problem. A systematic procedure is then introduced to solve this design problem. Second, experiments have been carried out with a direct impact system on the high-strength Ti-6Al-4 V titanium alloy at three strain rates until about $2 \cdot 10^{4} \mathrm{~s}^{-1}$ (till about $1.1 \cdot 10^{4} \mathrm{~s}^{-1}$ of plastic strain rate). Quasi-static experiments have also been carried out on the same alloy. In the third section, experimental data and the post-processing associated are presented and discussed. Third, the Johnson-Cook parameters are identified in the fourth section on experimental results, using in particular for the rate-dependent part of the model an inverse analysis coupled with the finite element code ABAQUS [27]. These data aim at feeding numerical simulations of dynamic material forming processes to make them more predictive.

\section{Design of the Direct-impact Configuration}

A direct-impact device consists of the projectile, the specimen, the transmitted bar (see Figure 1) and accessories such as the canon and the buffering device. Designing a dedicated experimental direct impact device comes down to the design of the bar, the specimen and the projectile and to determine its impact velocity. This leads to two topics classified by Ramesh [8] as the system design and the experimental design. The system design involves the determination of three important ratios, independent to the specific experiment carried out: the ratios of diameter to length of the transmitted bar $\left(\phi_{b} / l_{b}\right)$ and the specimen $\left(\phi_{s} / l_{s}\right)$, and the ratio of the specimen diameter to the bar diameter $\left(\phi_{s} / \phi_{b}\right)$. These ratios will be referred in the sequel to as the first, second and third ratios of the system design, respectively. The indices $(p, s, b)$ will refer in the sequel to the projectile, the specimen and the transmitted bar, respectively. The experimental design determines the specimen dimensions $\left(l_{s}, \phi_{s}\right)$, the length $\left(l_{p}\right)$ and the impact velocity $\left(v_{p}\right)$ of the projectile, to deform the specimen in such a way that a given strain rate be reached at a given level of strain.

\section{System design}

The three ratios of the system design aim primarily to ensure the unidimensional propagation of the loading wave within the device, while ensuring a sufficient strength to support the loading pulse. This condition is of primary importance for the identification of the specimen behaviour in this test. The diameter of the transmitted bar $\phi_{b}$ should be large enough to withstand the loading pulse without buckling or being plastically compressed. However, the errors in the stress-strain curve identification induced by dispersion and lateral effects become more important as the diameter increases. A first ratio far smaller than one

$\frac{\phi_{b}}{l_{b}} \leq \frac{1}{100}$

has been found acceptable [8]. Similarly, a reasonable range should be given to the second ratio $\phi_{s} / l_{s}$ to avoid buckling and to reduce the friction by decreasing the contact cross-section

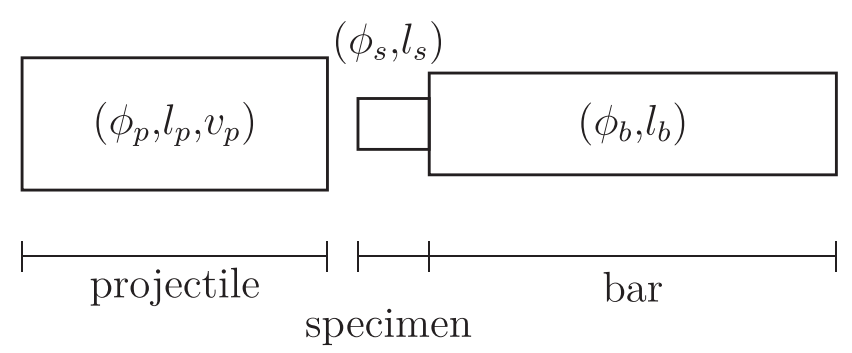

Figure I: Geometric schema of direct-impact Hopkinson 
areas at the interfaces between the specimen, the bar and the projectile. Ramesh [8] recommends it to be framed as follows:

$0.6 \leq \frac{\phi_{s}}{l_{s}} \leq 1$

For tests performed at very high strain rates on highstrength metallic materials, a wider range of $0.5-2$ is also acceptable [20]. The specimen length influences strongly the strain rate achieved. Indeed, because the strain rate is inversely proportional to the specimen length $l_{s}$, a shorter one increases the rate:

$\dot{\varepsilon}_{s}=\frac{v_{s}^{R}-v_{s}^{L}}{l_{s}}$

where $v_{s}^{L}$ and $v_{s}^{R}$ stand for the longitudinal velocities of the left and right cross-sections of the specimen, respectively.

A small value of the third ratio $\phi_{s} / \phi_{b}$ enables to ensure a good contact at the specimen/bar interface even if $\phi_{s}$ dilates largely during the plastic compression of the specimen, and allows then to test much more ductile materials. But the reduction of the specimen diameter is not unbounded. Safa and Gary [28] pointed out that impacting the bar with a very small-diameter specimen could increase the punching problem on the bar end. They also proposed an analytical approach to correct this punching effect [28]. The following empirical range

$\frac{1}{4} \leq \frac{\phi_{s}}{\phi_{b}} \leq \frac{1}{2}$

is usually adopted $[8,20]$.

\section{Experimental design}

The achievable strain rate is related to the dimensions of the specimen, these of the projectile and its impact velocity. When the projectile impacts the specimen, a loading pulse is generated so that two waves propagate simultaneously in opposite directions within the specimen and the projectile. In the projectile, the first wave propagates to the free end and is then reflected back to the impacting interface. Meanwhile, the second wave propagates through the specimen and is both reflected and transmitted to the transmitted bar. When the first wave, reflected at the end side of the projectile, arrives at the projectile/specimen interface at time $t=2 l_{p} / c_{p}$, referred to as the characteristic time, the impact is considered to be terminated. In this definition, $c_{p}$ denotes the sound speed in the projectile. In order to achieve a high strain rate $\dot{\varepsilon}_{S}$ in the specimen, the projectile should be accelerated to a sufficient velocity $v_{p}$ to deform the specimen, although the capacity of the canon may limit it. The engineering strain in the specimen can be estimated by $\varepsilon_{s}^{t} \approx \int_{0}^{2 l_{p} / c_{p}} \dot{\varepsilon} d t$

The experimental design has thus to simultaneously consider the expected strain rate and the allowable strain in the specimen.

\section{Additional design criteria}

Although the combination of the system design and the experimental design allows to restrict the range of possibilities for the design, some other constraints need to be fulfilled to complete the design. Moreover, the three ratios of the system design have been framed so far by empirical bounds. These additional constraints may clarify some of these empirical bounds.

First of all, the level of strain achieved within the specimen should be bounded. A sufficient level of strain $\varepsilon_{S_{\min }}$ is required to characterize correctly the dynamic behaviour of the material, whereas a maximum strain $\varepsilon_{S_{\max }}$ is required to avoid to crush the specimen. Two criteria are then involved. First, bounds on the length of the projectile $l_{p}$ can be assessed assuming a given average strain rate $\dot{\varepsilon}_{s_{\text {avg }}}$ during the characteristic time $t=2 l_{p} / c_{p}$ :

$\frac{c_{p}}{2}\left|\frac{\varepsilon_{S_{\min }}}{\dot{\varepsilon}_{S_{\text {avg }}}}\right| \leq l_{p} \leq \frac{c_{p}}{2}\left|\frac{\varepsilon_{S_{\max }}}{\dot{\dot{s}}_{S_{\text {avg }}}}\right|$

Second, an approximative upper bound of the impact velocity of the projectile can be assessed in order to avoid to exceed the allowable level of strain. Writing the conservation of energy applied to a system that consists of the projectile plus the specimen between their impact and depart times, and assuming a unidimensional system, a rigid projectile, a rigid perfectly plastic behaviour of the specimen and a vanishing velocity of the projectile at the end time, the impact velocity $v_{p}$ can be bounded as

$v_{p} \leq \sqrt{\frac{2 \sigma_{y_{s}}\left|\varepsilon_{s_{\max }}\right| l_{s} S_{s}}{\rho_{p} S_{p} l_{p}}}$

where $\sigma_{y_{s}}$ is the yield stress of the specimen, $l_{s}$ and $S_{s}$ are the length and the cross-section of the specimen, and $\rho_{p}, S_{p}$ and $l_{p}$ are the mass density, the cross-section and the length of the projectile, respectively. Of course, a refined bound could be assessed using a more complex constitutive model to compute the strain energy of the specimen, and considering the projectile as deformable.

Then, although the bar should remain elastic during the test, it has to be sufficiently strained to record a usable signal in post-processing. On the one hand, strength criteria of the bar pertain to its resistance to buckling and to plasticity. The former can be assessed in a first approximation through the critical load obtained in quasi-static: 
$F_{b} \leq \frac{\pi^{2} E_{b} I_{b}}{L^{2}}$

where $F_{b}$ denotes the force in the bar, $E_{b}$ its Young's modulus, $L$ is the length between two supports, equal to $l_{b} / 2$ if three supports are used and $I_{b}$ is the inertia moment about the bar axis. The latter combined with the force equilibrium assumed to be achieved within the specimen enables to give an upper bound to the third ratio of the system design:

$\frac{\phi_{s}}{\phi_{b}} \leq \sqrt{\frac{\sigma_{y_{b}}}{\alpha\left|\sigma_{s_{\max }}\right|}}$

where $\alpha$ denotes a safety factor (greater than one) and $\sigma_{s_{\max }}$ refers to an expected maximum level of stress within the specimen. On the other hand, a usable signal for post-processing can be roughly assessed so that the force in the bar becomes more than one third of the yield force in the specimen, in order to pass over the noise in the measurements during the beginning of the deformation process. Provided a minimum level of strain recorded by gauges on the bar $\varepsilon_{b_{\text {min }}}$, another upper bound to the third ratio of system design can be given:

$\frac{\phi_{s}}{\phi_{b}} \leq \sqrt{\frac{3 E_{b}\left|\varepsilon_{b_{\min }}\right|}{\sigma_{y_{s}}}}$

Afterwards, because the test is assumed to be terminated at the end of the characteristic time, the force equilibrium within the specimen should be reached before this time, so that its writing can be used in the post-processing to extract directly the stress-strain curve a posteriori. It is generally considered that a great number of round trips of the wave within the specimen should be achieved during the characteristic time:

$\frac{2 l_{s}}{c_{s}} \ll \frac{2 l_{p}}{c_{p}}$

The length of the bar has to be designed so that on the one hand a unidimensional propagation of the wave is ensured, which requires a minimum slenderness; the length should be at least 10 times the diameter. On the other hand, no wave reflection should occur at the free end of the bar during the characteristic time. Combining both items, one obtains

$l_{b} \geq \frac{2 l_{p} c_{b}}{c_{p}}+10 \phi_{b}$

This last inequality comes in addition to that of the first ratio of the system design that couples the length and the diameter of the bar.
Finally, the diameter of the projectile has to be bounded from above by that of the canon:

$\phi_{p} \leq \phi_{\text {canon }}$

\section{Diameter of the transmitted bar}

The loading pulse propagates in the transmitted bar as a plane wave, which consists of a superposition of modes. If we want to compute directly the stress in the bar from the recorded strain, we have to make sure that the sole first mode of the bar will be excited by the loading pulse. Thus, the profile of this loading signal has first to be assessed. Second, the spectrum of the bar is needed, and more precisely, the frequency of the second mode. The well-known Pochhammer-Chree [29, 30] analytical solution enables to relate the radius of the bar to the angular frequency of a given mode. Determining an upper bound for the bar diameter thus comes down to compare the cut-off frequency of the exciting signal with respect to the frequency of the second mode of the bar. In order to assess the profile of the loading signal that propagates within the bar, a constitutive model can be postulated to describe the behaviour of the specimen, and therefore to assess its response to the initial pulse. The cut-off frequency of this signal is then computed in the frequency space through a Fourier transform. The Johnson-Cook model has here been used in a first approximation with parameters calibrated for the Ti- $6 \mathrm{Al}-4 \mathrm{~V}$ alloy at the strain rate of $20 \mathrm{~s}^{-1}$ [24]; the plotted stress-strain curve is converted into time space assuming a constant strain rate of $10^{5} \mathrm{~s}^{-1}$ and a strain ranging from 0 to 0.5 . The profile of the exciting signal is plotted in Figure 2.

The Pochhammer-Chree solution is obtained by solving the set of elastodynamic equations for an infinite cylinder. Non-trivial solutions are given when the PochhammerChree equation (Equation [14]) [29-31] vanishes:

$$
\begin{aligned}
\phi\left(\xi, \omega, E, v, r_{b}, \rho\right)= & \frac{2 \alpha}{r_{b}}\left(\beta^{2}+\xi^{2}\right) J_{1}\left(\alpha r_{b}\right) J_{1}\left(\beta r_{b}\right) \\
& -\left(\beta^{2}-\xi^{2}\right)^{2} J_{0}\left(\alpha r_{b}\right) J_{1}\left(\beta r_{b}\right) \\
& -4 \alpha \beta \xi^{2} J_{1}\left(\alpha r_{b}\right) J_{0}\left(\beta r_{b}\right)=0
\end{aligned}
$$

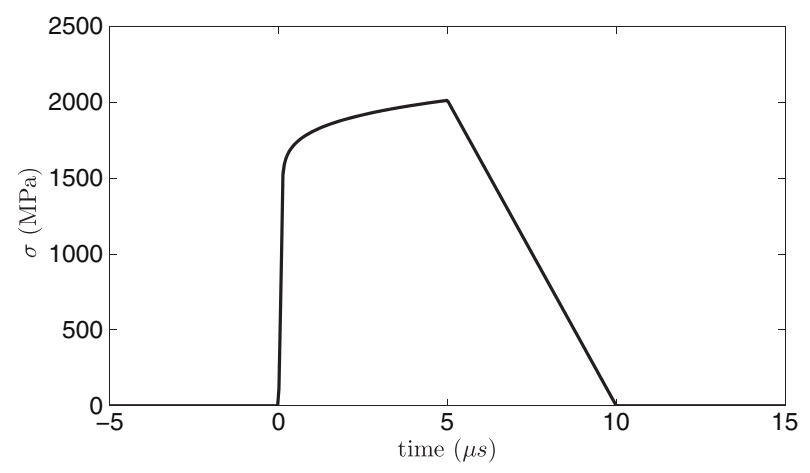

Figure 2: Exciting signal 
where $\alpha^{2}=\frac{\rho \omega^{2}}{\lambda+2 \mu}-\xi^{2}$ and $\beta^{2}=\frac{\rho \omega^{2}}{\mu}-\xi^{2}, J_{n}(\cdot)$ is the Bessel function of the first kind at order $n, \xi$ is the wave number, $\omega$ is the angular frequency, $r_{b}$ is the bar radius, and $v, \lambda, \mu, E, \rho$ denote the Poisson's ratio, the Lamé's constants, the Young's modulus and the mass density, respectively. This equation gives an implicit relation between the wave number, the angular frequency, the bar radius and the material properties of the bar. The limit angular frequencies of the modes of an elastic bar are given for a vanishing wave number:

$\xi\left(\omega_{l}^{(m)}\right)=0$

where $m$ refers to the mode number. Thus, we obtain an equation satisfied by the limit frequencies. The solution of the obtained equation allows to relate the angular frequency of the second mode to the radius of the bar, as plotted for a classical steel in Figure 3. The superposed horizontal line refers to the cut-off frequency $\omega_{c}$ of the loading signal. In other words, any higher frequency than this cut-off frequency will not be excited during the test. Figure 3 gives an upper bound for the radius of about $r_{b}=9 \mathrm{~mm}$, so that the sole first mode will be excited.

\section{An optimization problem}

Provided some input data, the design problem of the directimpact configuration can be formulated as an optimization problem submitted to equality and inequality constraints.

First, we assume to be given a family of materials we want to test at a given objective strain rate $\dot{\varepsilon}_{s_{\mathrm{obj}}}$, so that the level of strain in the specimen be framed between its given minimum $\varepsilon_{S_{\min }}$ and maximum $\varepsilon_{S_{\max }}$ values, and so that a minimum level of recorded strain $\varepsilon_{b_{\min }}$ be reached. Second, according to the family of material to be tested, we assume to be chosen the material of the projectile and the bar, so that their Young's modulus and mass density (and thus their sound speed), and the yield stress of the bar $\sigma_{y b}$ be known. Third, the canon is assumed to be available; thus, the constraint (Equation [13])

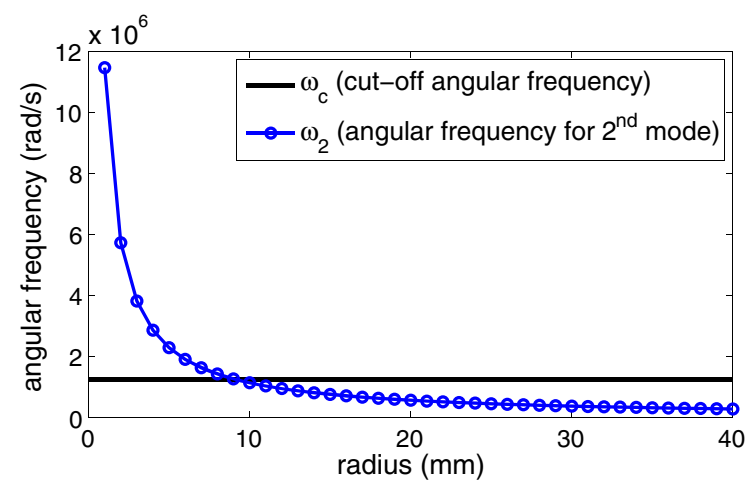

Figure 3: Cut-off frequency $\omega_{c}$ of the loading pulse and angular frequency of the second mode of the bar determines a priori the diameter of the projectile $\phi_{p}$ provided a given diametral clearance $\epsilon$. Finally, we assume to be given at least a coarse constitutive model of the specimen material in order to be able to assess the yield stress $\sigma_{y_{s}}$, the maximum level of stress reached $\sigma_{s_{\max }}$ and the sound speed $c_{s}$.

The unknown vector $\mathbf{x}$ associated to the optimization problem consists of the length $l_{p}$ of the projectile and its impact velocity $v_{p}$, the dimensions of the specimen $\left(l_{s}, \phi_{s}\right)$ and these of the bar $\left(l_{b}, \phi_{b}\right)$ :

$\mathbf{x}=\left\{l_{p}, v_{p} ; \phi_{s}, l_{s} ; \phi_{b}, l_{b}\right\}$

A solution of $\mathbf{x}$ may be sought by comparing the computed value of the strain rate $\dot{\varepsilon}_{s}(\mathbf{x})$ during the design process to the objective one $\dot{\varepsilon}_{S_{\mathrm{obj}}}$. The cost function is thus defined as follows:

$f(\mathbf{x})=\left|\dot{\varepsilon}_{S}(\mathbf{x})-\dot{\varepsilon}_{S_{\mathrm{obj}}}\right|$

where the strain rate $\dot{\varepsilon}_{S}$ within the specimen is computed in the case of identical material but different diameters of the projectile and the bar by the formulae:

$\dot{\varepsilon_{s}}=-\frac{v_{p}+\frac{S_{p}+S_{b}}{S_{p}} C_{b} \varepsilon_{b}(t)}{l_{s}}$

that will be demonstrated later on in the section pertaining to the post-processing, where $S_{p}$ and $S_{b}$ are the cross-sections of the projectile and the bar, respectively. The strain $\varepsilon_{b}$ in the bar is related to the stress in the specimen and to the second ratio of the system design through the equilibrium of the specimen/bar interface:

$\varepsilon_{b}=\frac{\sigma_{s}}{E_{b}}\left(\frac{\phi_{s}}{\phi_{b}}\right)^{2}$

The optimization problem submitted to equality and inequality constraints is thus formulated as follows:

$\min _{\mathbf{x}} f(\mathbf{x}) ; \quad \mathbf{G}(\mathbf{x}) \leq 0 ; \quad \mathbf{h}(\mathbf{x})=0$

where $\mathbf{h}(\mathbf{x})=0$ is a set of equality constraints that consists of Equations [18] and [19]. The set of inequality constraints $\mathbf{G}$ $(\mathbf{x}) \leq 0$ consists of inequalities [1], [2], [4], [6], [7], [8], [9], [10], [11], [12], and is added the upper bound prescribed on the bar diameter given in the previous section summarized through the following implicit relation:

$g\left(\phi_{b}, \omega_{c}, \omega_{b}^{(2)}\right) \leq 0$

where $\phi_{b}, \omega_{c}$ and $\omega_{b}^{(2)}$ denote the bar diameter, the cut-off frequency of the loading pulse and the angular frequency 
of the second mode of the bar, respectively. Notice also that the cost function defined by Equation [17] may a priori exhibit several minima.

\section{Design procedure}

The problem (Equation [20]) has to be solved iteratively. Actually, it appears that the length and the diameter of the specimen $\left(l_{s}, \phi_{s}\right)$, and the length of the projectile $l_{p}$ play the role of driving unknowns within the solution procedure because they are framed between lower and upper bounds. Indeed, the other components of the unknown vector $\mathbf{x}$ and the cost function (Equation [17]) can be computed directly from these three first ones.

The procedure starts by computing the upper bound of the bar diameter from the loading pulse cut-off frequency, assessed from given data associated to the family of material we want to test. Three nested loops defined on the three driving unknowns run the iterative process within their computed bounds. The other components of the unknown vector $\mathbf{x}$ and the cost function are then directly computed provided some conditions are satisfied. The process is stopped when the cost function becomes smaller than a given tolerance TOL. The flowchart of the design procedure of the direct-impact system is summarized in Algorithm 1.

\section{Algorithm 1 Design procedure of the direct-impact system}

1: Assess $\omega_{c}$ of the loading pulse computed with $\dot{\varepsilon}_{s_{\mathrm{obj}}}$ and $\varepsilon_{s_{\max }}$.

2: Deduce $\phi_{b_{\max }}$ from [21] provided a given safety factor.

3: if $\phi_{p}=\phi_{\text {canon }}-\boldsymbol{\epsilon}$ then

4: Compute $\phi_{s_{\min }}$ and $\phi_{s_{\max }}$ satisfying to [4], [9] and [10].

5: Compute $l_{p_{\min }}$ and $l_{p_{\max }}$ satisfying to [6].

6: for $\phi_{s} \in\left[\phi_{s_{\min }}, \phi_{s_{\max }}\right] \mathbf{d o}$

7: Compute $l_{s_{\min }}$ and $l_{s_{\max }}$ satisfying to [2].

8: $\quad$ for $l_{s} \in\left[l_{s_{\min }}, l_{s_{\max }}\right]$ do

9: $\quad$ for $l_{p} \in\left[l_{p_{\min }}, l_{p_{\max }}\right]$ do

10: $\quad$ if $l_{p}$ satisfies [11] then

11: $\quad$ Compute $v_{p}$ satisfying to [7].

12: $\quad$ Compute $l_{b}$ satisfying to [1] and [12].

13: $\quad$ Compute $\varepsilon_{b}$ with [19].

14: $\quad$ if $\varepsilon_{b} \geq \varepsilon_{b_{\min }}$ and [8] is satisfied then

15: $\quad$ Compute $\dot{\varepsilon}_{s}$ with [18].

16: $\quad$ Compute the cost function [17]

17: $\quad$ if $f(\mathbf{x}) \leq$ TOL then

18: $\quad$ Break;

19: $\quad$ end if

20: $\quad$ end if

21: end if

22: end for

23: end for

24: end for

25: end if

\section{Experiments}

\section{Designed direct-impact device}

The direct-impact device designed to perform the dynamic test is shown in Figure 4 . It consists of a transmitted bar of $1.2 \mathrm{~m}$ length and $10 \mathrm{~mm}$ diameter, and a projectile of $15.8 \mathrm{~mm}$ diameter, according to the inner diameter of the canon. An absorbing bar and a buffering device are also used to damp the excess energy. The two bars and the projectile are made of a high-strength steel (MARVAL X2NiCoMo18-8-5) with a yield stress of $1800 \mathrm{MPa}$. Three supports equipped with bushings made of teflon guide the transmitted bar. The bar is instrumented with three bridges with double strain gauges mounted in opposition on the bar, located at the distances of 150,600 and $1080 \mathrm{~mm}$ away from the specimen/transmitted bar interface. The three bridges allow to monitor if dispersion occurs along the bar. The first one is used for the postprocessing. The specimen is lubricated on both end-sections with some grease to decrease the friction.

\section{Experimental plan}

The specimen length, the projectile length and its impact velocity are the three parameters that need to be designed to achieve an expected strain rate within the specimen. However, the combination of these three parameters is not unique to have a given strain rate. Moreover, provided a given maximum energy supplied by the canon to the projectile, its impact velocity can be directly deduced from its length.

Consequently, only two parameters are independent. The greater the impact velocity and the smaller the length of the specimen, the greater strain rate can be achieved. But the available range of these two parameters must remain bounded in order to avoid to crush the specimen. In Table 1, three combinations of these two parameters are designed to reach three expected strain rates ranging from $4 \cdot 10^{3}$ to $20 \cdot 10^{3} \mathrm{~s}^{-1}$.

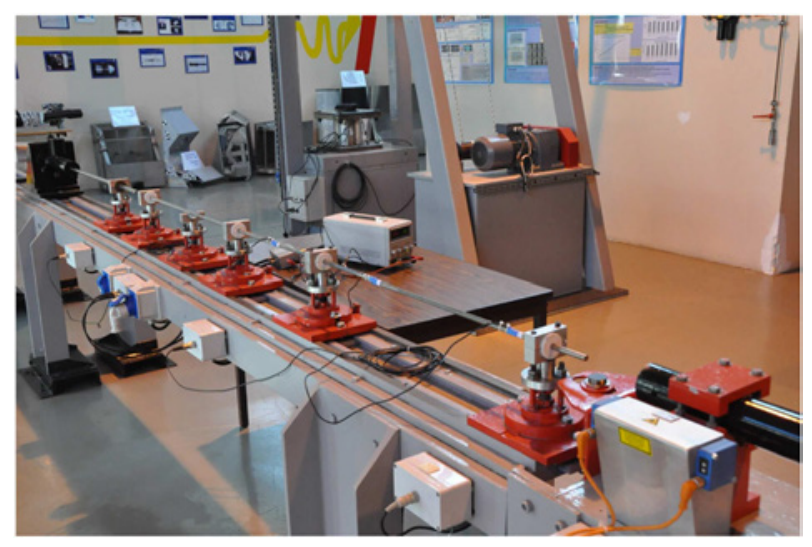

Figure 4: Direct-impact device 
Table I: Experimental plan

\begin{tabular}{lccccc}
\hline & $\begin{array}{c}\text { Expected } \\
\varepsilon\left(\mathrm{s}^{-1}\right)\end{array}$ & $v_{p}\left(\mathrm{~ms}^{-1}\right)$ & $I_{p}(\mathrm{~mm})$ & $I_{s}(\mathrm{~mm})$ & Max. $\dot{\varepsilon}\left(\mathrm{s}^{-1}\right)$ \\
\hline Test no. & 4000 & 23.2 & 125 & 4.93 & 4642 \\
2 & 10000 & 32.2 & 60 & 3.04 & 10740 \\
3 & 20000 & 29.4 & 60 & 1.60 & 18350 \\
\hline
\end{tabular}

Besides, quasi-static tests in tension have also been carried out at the strain rate of $10^{-4} \mathrm{~s}^{-1}$ on an Instron 5580 load frame test machine, to obtain the material flow curve under quasi-static loading.

\section{Experimental results}

First of all, the quasi-static tensile flow curve obtained from the traction machine is depicted in Figure 5. Second, Figure 6 shows the strains $\varepsilon_{b}(t)$ recorded on the bar for the three experiments performed with the direct impact Hopkinson system. The profile of these strain curves are close to these usually observed with the classical SHPB, except that few trays appear during the discharge, especially on the first test while they are rather guessed on the two others. This is due to the unloading waves reflected at the free end of the projectile. Indeed, because the length of the projectile has been decreased with respect to that of the classical SHPB in order to increase its impact velocity, the characteristic time associated to a round trip of the wave has been decreased and is clearly seen in the discharge regime of the recorded strain curves.

\section{Post-processing}

The common and main assumption usually performed in the post-processing [9-20] is to consider the force equilibrium achieved within the specimen. The stress is then given by

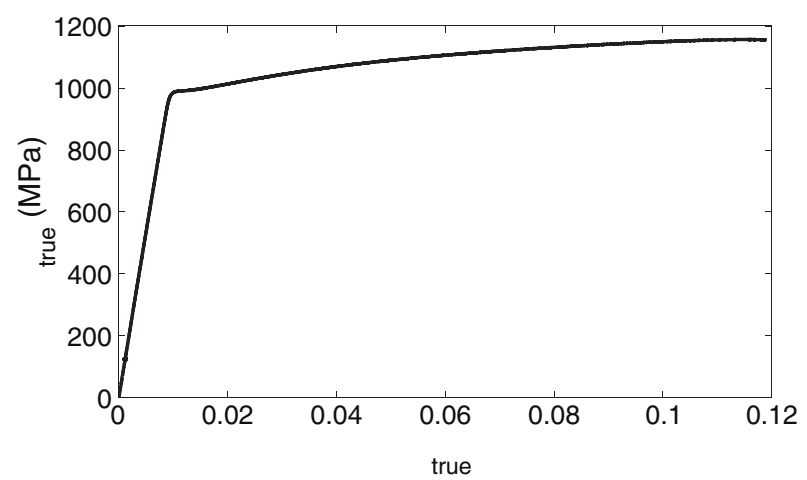

Figure 5: Quasi-static tensile stress-strain curve

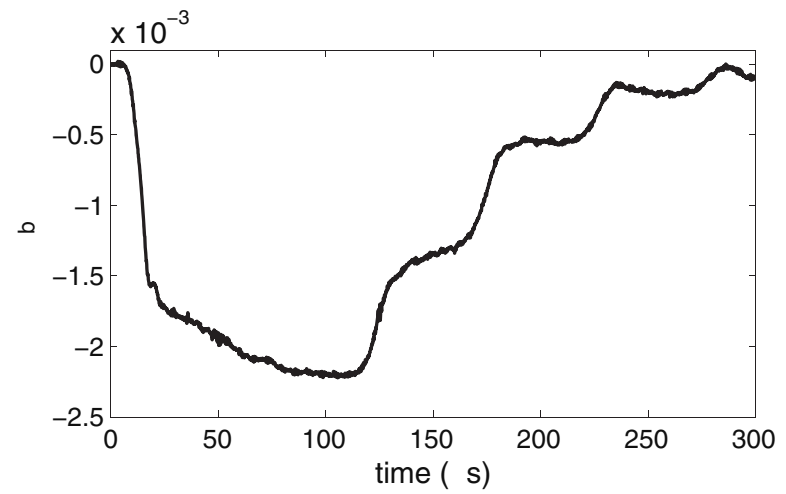

(a) Test 1

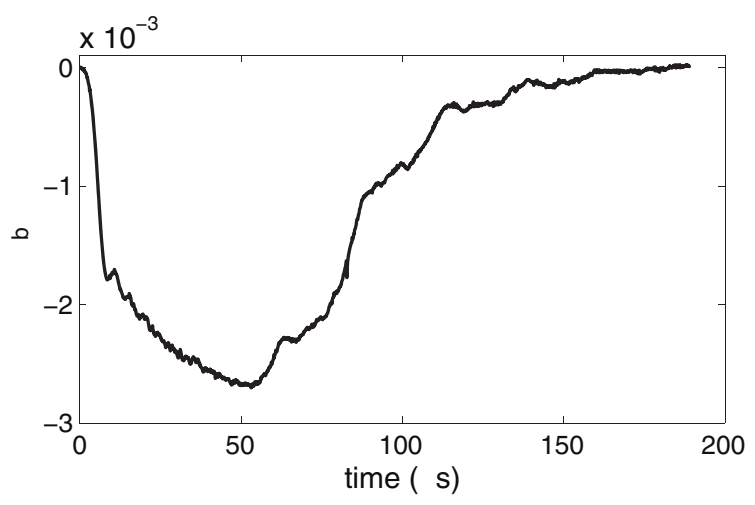

(b) Test 2

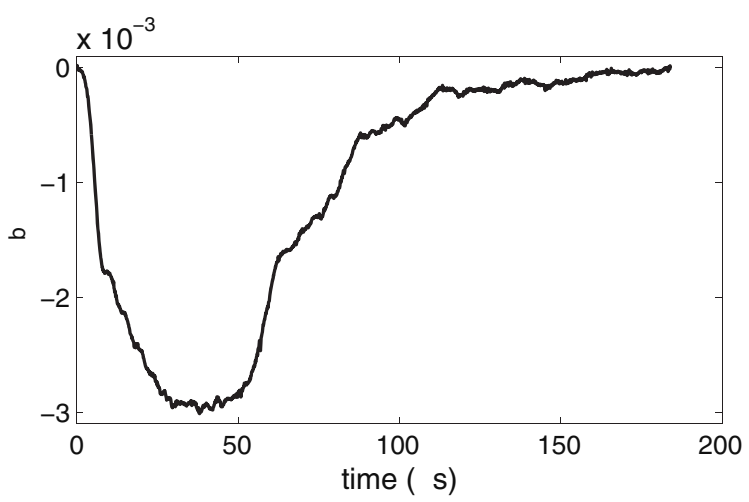

(c) Test 3

Figure 6: Recorded strain for the three tests

$\sigma_{s}(t)=\frac{S_{b} E_{b}}{S_{s}} \varepsilon_{b}(t)$

where $\varepsilon_{b}(t), S_{b}$ and $S_{s}$ denote the strain recorded on the bar, and the cross-sections of the projectile and the specimen, respectively.

Without any incident bar as used in the classical SHPB, the calculation of the strain within the specimen requires extra measurements and/or computations. Several approaches have been followed so far to this end. Gorham [9] developed an optical system to record the deformation of the specimen 
using a high-speed camera. Malinowski et al. [32] measured the displacement $U_{1}(t)$ at the projectile/specimen interface, and expressed the displacement at the specimen/bar interface as

$U_{2}(t)=-\int_{0}^{t} c_{s} \varepsilon_{b}(\tau) d \tau$

where $c_{s}$ denotes the sound speed in the specimen, $\varepsilon_{b}(t)$ is negative in the compression test. The strain in the specimen is thus given by [32]

$\varepsilon_{s}(t)=-\frac{1}{l_{s}}\left[U_{1}(t)+c_{s} \int_{0}^{t} \varepsilon_{b}(\tau) d \tau\right]$

Assuming the force equilibrium achieved within the specimen, the strain is computed as [1]

$\varepsilon_{s}(t)=-\frac{1}{l_{s}}\left[v_{p} t+\frac{\left(Z_{p}+Z_{b}\right)}{Z_{p} Z_{b}} E_{b} \int_{0}^{t} \varepsilon_{b}(\tau) d \tau\right]$

where $v_{p}$ is the velocity of the projectile, $Z_{p}$ and $Z_{b}$ stand for the impedance $\left(Z_{i}=\rho_{i} c_{i}, i \in\{p, b\}\right)$ of the projectile and the bar, and $E_{b}$ is the Young's modulus of the bar.

In this work, because the projectile and the bar have different cross-sections, the following approach is used. The velocity $v_{s}^{R}$ at the specimen/bar interface is expressed as a function of the recorded strain $\varepsilon_{b}(t)$ :

$v_{s}^{R}=-c_{b} \varepsilon_{b}(t)$

where $c_{b}$ is the sound speed in the bar. The velocity at the projectile/specimen interface $v_{s}^{L}$ is in turn given by

$v_{s}^{L}=v_{p}+c_{p} \varepsilon_{p}$

where $\varepsilon_{p}$ refers to the strain in the projectile, and $c_{p}$ its sound speed, equals to that of the bar $\left(c_{b}=c_{p}\right)$ provided both are made of the same material. The equilibrium then reads

$E_{b} \varepsilon_{b}(t) S_{b}=E_{p} \varepsilon_{p} S_{p}$

where $S_{b}$ and $S_{p}$ are the cross-sections of the transmitted bar and the projectile, and $E_{p}$ and $E_{b}$ are the (identical, $E_{p}=E_{b}$ ) Young's modulus of the projectile and the transmitted bar. Combining Equations [27] and [28], the strain in the specimen is computed by the following equation:

$$
\begin{aligned}
\varepsilon_{s}(t) & =\int_{0}^{t} \frac{v_{s}^{R}-v_{s}^{L}}{l_{s}} d \tau \\
& =-\frac{1}{l_{s}}\left[v_{p} t+\frac{S_{b}+S_{p}}{S_{p}} c_{b} \int_{0}^{t} \varepsilon_{b}(\tau) d \tau\right]
\end{aligned}
$$

The strain rate is computed by taking the time derivative of Equation [29] and yields
$\dot{\varepsilon_{S}}=-\frac{v_{p}+\frac{S_{p}+S_{b}}{S_{p}} c_{b} \varepsilon_{b}(t)}{l_{S}}$

thereby find Equation [18]. Equations [29] and [30] are valid only before the end of the loading, as Gorham [1] pointed out. The maximal strain rates computed with Equation [30] reached during the tests are listed in Table 1.

Because the large strain regime is reached during the impact, the engineering stress and strain expressions computed by Equations [22] and [29] are converted to the true stress and strain measures by the classical formula:

$\varepsilon_{\text {true }}=\ln \left(1+\varepsilon_{\text {eng }}\right)$

$\sigma_{\text {true }}=\sigma_{\text {eng }}\left(1+\varepsilon_{\text {eng }}\right)$

The true stress-strain curves are plotted in Figure 7. In Figure $7(\mathrm{~B}$ and $\mathrm{C})$, remarkable oscillations arise in the elastic-plastic part of the curve. These oscillations may be associated to the increasing number of reflections of the wave within the specimen due to the very small initial length of the specimen and to its even smaller length at the peak compression of the strain. Consequently, some difficulties arise in the classical post-processing when higher strain rates are aimed to be achieved.

The reliability of these curves and their representativeness of the behaviour of the titanium alloy at these strain rates can be thought flawed. Indeed, it is difficult to keep the assumption of force equilibrium in the specimen for at least two reasons. First, as the achieved strain rate increases, inertia quantities take a greater importance. Second, the writing of the force equilibrium (Equation [22]) leads to a huge uncertainty on the elastic part of the computed stress-strain curve, and thus on the assessment of the elastic stiffness and the yield stress.

Consequently, a dynamic analysis has to be carried out to account for inertia quantities. Thus, the computation of the stress-strain curve is not direct anymore, and an inverse analysis involving a direct numerical analysis is needed. A constitutive model is postulated to describe the behaviour of the material tested. The parameters of this constitutive model are then identified so that some given quantities extracted from the numerical simulation fit experimental data.

\section{Inverse Analysis: Constitutive Model Identification}

\section{Johnson-Cook constitutive model}

Among the available constitutive model describing the rate dependence of metallic materials, the Johnson-Cook model [23] is one of the most popular because of its simplicity, and is widely used to model the behaviour of metallic materials in dynamic conditions. It consists of an empirical expression of the flow stress $\sigma_{y}$ that accounts for the strain hardening, 


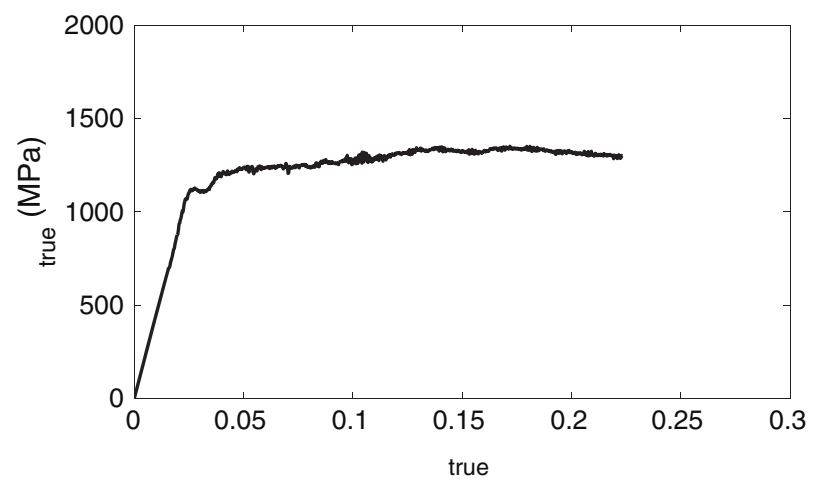

(A) Test 1

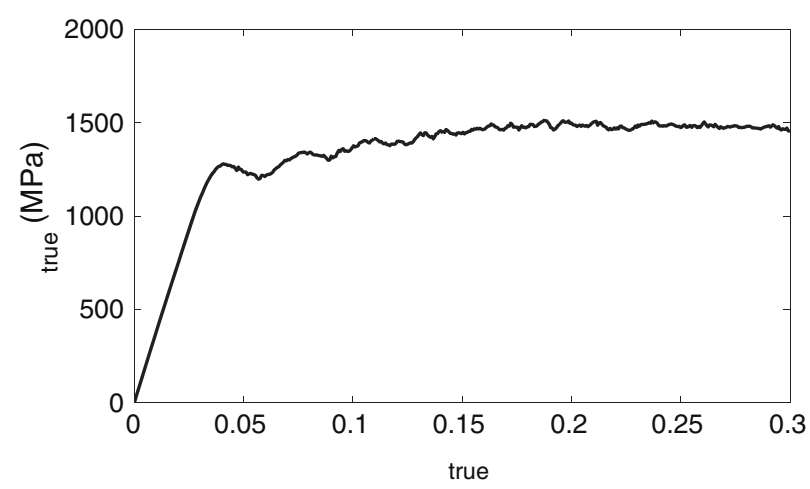

(B) Test 2

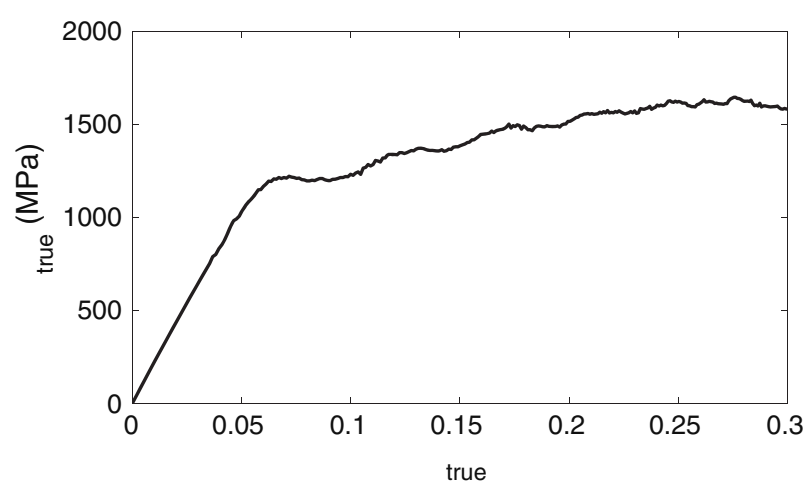

(C) Test 3

Figure 7: Stress-strain curves

strain rate and temperature effects through a reduced number of parameters, which allows for an easier identification. It reads

$$
\begin{aligned}
\sigma_{y}\left(\varepsilon_{\mathrm{eq}}^{p}, \dot{\varepsilon}_{\mathrm{eq}}^{p}, T\right)= & \left(A+B\left(\varepsilon_{\mathrm{eq}}^{p}\right)^{n}\right) \\
& \left.\times 1+C \ln \left(\frac{\dot{\varepsilon}_{\mathrm{eq}}^{p}}{\dot{\varepsilon}_{0}}\right)\right)\left(1-\left(\frac{T-T_{\text {ref }}}{T_{\text {melt }}-T_{\text {ref }}}\right)^{m}\right)
\end{aligned}
$$

where $A, B, C, n$ and $m$ are the material parameters, $\dot{\varepsilon}_{0}$ is the reference strain rate, $T_{\text {ref }}$ and $T_{\text {melt }}$ are the reference and melting temperatures respectively, and $\varepsilon_{\mathrm{eq}}^{p}$ and $\dot{\varepsilon}_{\mathrm{eq}}^{p}$ stand for the equivalent (cumulated) plastic strain and the equivalent strain rate.

\section{Identification}

In this work, only the strain hardening and rate-dependence effects are studied; therefore, thermal effects are not addressed here. First of all, the parameters $A, B$ and $n$ are identified on the quasi-static tensile flow curve shown in Figure 5. The identification is performed by integrating the elastic-plastic constitutive model [33] with an isotropic hardening given by the first parenthesis of Equation [33] using a least square fit. The identified values of these parameters are summarized in Table 2.

Second, the identification of the parameter $C$ requires to perform an inverse analysis involving a dynamic numerical computation carried out with the finite element code ABAQUS [27]. This parameter is ascribed to vary within reasonable bounds $(0.005 \leq C \leq 0.05)$ given according to the reference identified parameters of the Johnson-Cook model for Ti-6Al-4 V in [24-26]. The identification is performed by minimizing in the least square sense the cost function $f($ ) computed with the Euclidean norm of the difference between the simulated strain $\varepsilon_{\text {sim }}(, t)$ and the recorded one $\varepsilon_{\exp }(t)$ over a given time duration:

$f(\quad)=\left\|\varepsilon_{\text {sim }}(, t)-\varepsilon_{\exp }(t)\right\|_{2}$

where $\varepsilon_{\text {sim }}(, t)$ is computed from a numerical dynamic analysis of the direct-impact system run with ABAQUS/Explicit accounting for the formerly identified parameters summarized in Table 2. A bidimensional axisymmetric model consisting of the projectile, the specimen and the transmitted bar is defined to perform the simulation, in which experimental dimensions of the device are used. A truncated view of the mesh used is shown in Figure 8. Unilateral and frictionless contact conditions

Table 2: Identified $A, B$ and $n$

\begin{tabular}{lccc}
\hline$A(\mathrm{MPa})$ & $B(\mathrm{MPa})$ & $n$ & $\varepsilon_{0}\left(\mathrm{~s}^{-1}\right)$ \\
\hline 955 & 770 & 0.557 & 0.0001 \\
\hline
\end{tabular}

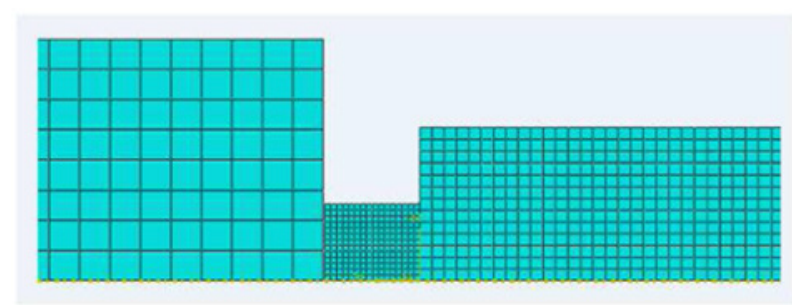

Figure 8: Zoom on the specimen of the bidimensional axisymmetric mesh of test 1 
are used at the projectile/specimen and specimen/bar interfaces. An initial condition is prescribed on the velocity of the projectile, set at its measured impact one during the experiments. The computed strain $\varepsilon_{\text {sim }}(t)$ is extracted from the numerical model at the same location than that of the first strain gauge stuck on the transmitted bar.

The numerical model consists of 22568 degrees of freedom. An element length of $0.167 \mathrm{~mm}$ is assigned to the specimen mesh; the smallest element length of the bar close

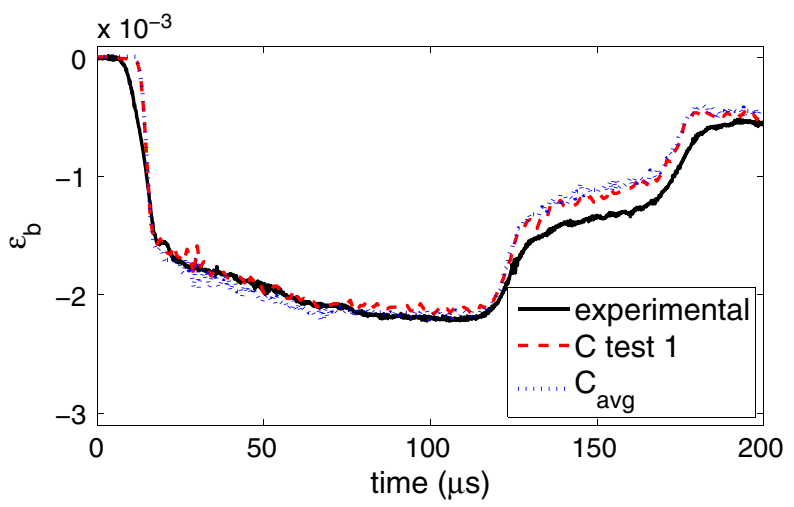

(A) Test 1

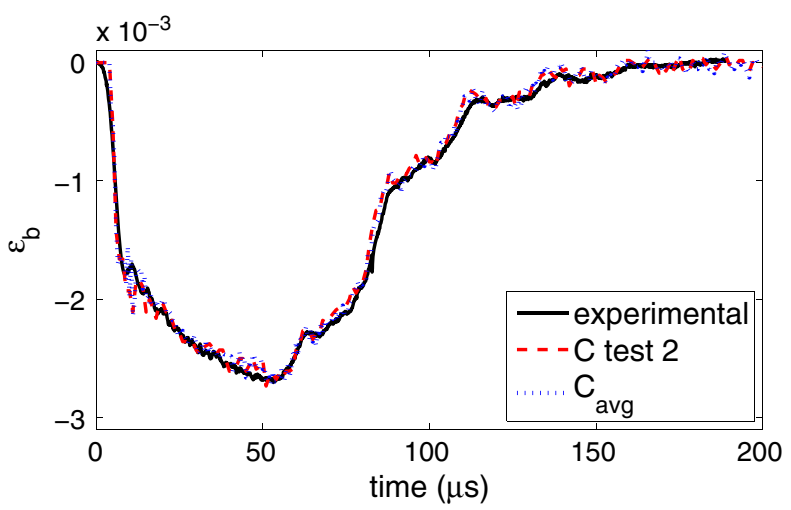

(B) Test 2

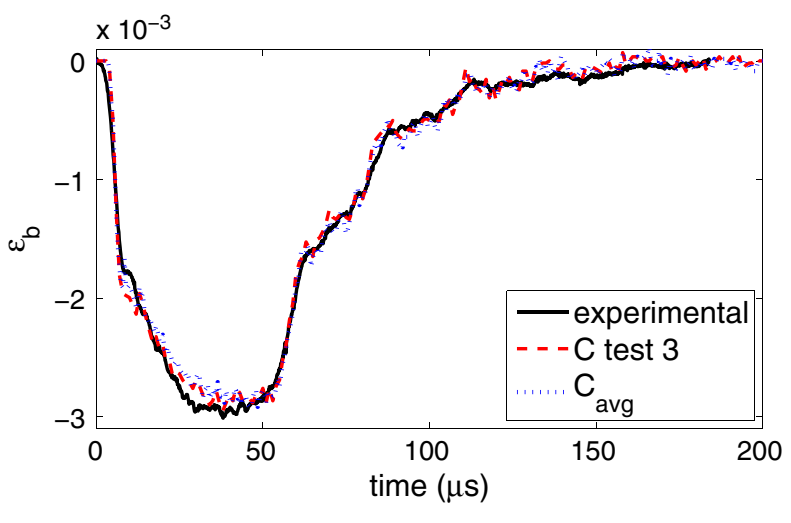

(C) Test 3

Figure 9: Recorded and calibrated strain curves
Table 3: Calibrated Johnson-Cook parameter $C$

\begin{tabular}{lcc}
\hline Test no. & $C$ & Max. $\dot{\varepsilon}_{\text {eq }}^{p}\left(s^{-1}\right)$ \\
\hline 1 & 0.009 & 2700 \\
2 & 0.013 & 7100 \\
3 & 0.015 & 11000 \\
\hline
\end{tabular}

to the specimen/bar interface is set to $0.38 \mathrm{~mm}$. The simulation is performed over a duration of $2 \cdot 10^{-4} \mathrm{~s}$ for the three tests.

\section{Results}

The computed strains resulting from the identification and the recorded strains are plotted and compared in Figure 9. The dashed curves refer to the computed strain, whereas the solid ones refer to the measurements. Although a small discrepancy between numerical and experimental curves is observed at the beginning of the first raise and in the unloading part of the first test, a good correlation between both curves is observed for the three tests; in particular, the reflected unloading waves are well simulated for the last two tests.

Table 3 lists the calibrated values of the parameter $C$ and the plastic strain rates $\dot{\varepsilon}_{\mathrm{eq}}^{p}$ derived from the simulations. The plastic strain rates in this table are smaller than the experimental ones in Table 1 because the maximum experimental strain rates are derived at the beginning of the impact when the elastic strain rates are maximum.

\section{Discussions}

The three values obtained for the parameter $C$ are close to each other; it is thus interesting to assess whether its variation has a significant influence on the rate behaviour of the specimen and hence on the strain curves. An average value $C_{\mathrm{avg}}=0.012$ is defined and used to perform three numerical simulations according to the three tests studied. As shown in Figure 9, only a minor difference exists between the dashed and the dotted curves. A constant value $C_{\text {avg }}$ can thus be used in the Johnson-Cook model to describe the behaviour of the Ti-6Al-4 $\mathrm{V}$ alloy within the range of strain rate $\dot{\varepsilon} \in[4500,18500] \mathrm{s}^{-1}$.

\section{Conclusion}

In this work, an inverse identification of the Johnson-Cook constitutive model has been performed on the titanium alloy Ti-6Al-4 $\mathrm{V}$ at three strain rates until about $2 \cdot 10^{4} \mathrm{~s}^{-1}$ (till about $1.1 \cdot 10^{4} \mathrm{~s}^{-1}$ of plastic strain rate) on a specially designed direct impact Kolsky bar device.

First, the design of the direct-impact configuration is reviewed and completed to provide a helpful systematic 
procedure. The design criteria required to ensure the accuracy of the measurements and calculations are initially introduced. The design of this device is shown to be the solution of an optimization problem submitted to equality and inequality constraints. A dedicated algorithm is then introduced to solve this problem.

Next, experiments have been carried out with a specially designed direct impact system on the high-strength Ti-6Al$4 \mathrm{~V}$ titanium alloy. Three particular tests reaching at three strain rates until about $2 \cdot 10^{4} \mathrm{~s}^{-1}$ (till about $1.1 \cdot 10^{4} \mathrm{~s}^{-1}$ of plastic strain rate) within the specimen are then studied. The estimated and experimentally computed strain rates are very similar, while the equivalent plastic strain rates from simulations are much lower, about one half. The reason is that the maximum values in Table 1 refer to an initial peak in the elastic region, whereas the major part of the loading history occurs at lower strain rates. Because the classical post-processing shows some spurious oscillations in the stress-strain curves on the tests achieving the higher strain rates, an inverse analysis coupled with the finite element code ABAQUS is carried out to identify the rate-dependent part of the Johnson-Cook model on these experiments, while the parameters $A, B$ and $n$ are identified on the quasi-static flow curve stress obtained experimentally. The differences between the values identified for the parameter $C$ on the different experiments are shown to have a small influence on the strain curves; hence, an average of the different values identified of this parameter is considered sufficient to describe the behaviour of the Ti-6Al-4 V titanium alloy within the range of strain rate $\dot{\varepsilon} \in[4500,18500] \mathrm{s}^{-1}$.

\section{REFERENCES}

1. Gorham, D. A., Pope, P. H. and Field, J. E. (1992) An improved method for compressive stress-strain measurements at very high strain rates. Compressive stress-strain measurements. Proc. R. Soc. Lond. A 438, 153-170.

2. Psyk, V., Risch, D., Kinsey, B. L., Tekkaya, A. E. and Kleiner, M. (2011) Electromagnetic Forming - a review. J. Mater. Process. Technol. 211, 787-829.

3. Meguid, S. A., Shagal, G. and Stranart, J. C. (1990) Finite element modelling of shot-peening residual stresses. J. Mater. Process. Technol. 92, 401-404.

4. Meguid, S. A., Shagal, G. and Stranart, J. C. (2002) 3D FE analysis of peening of strain-rate sensitive materials using multiple impingement model. Int. J. Impact. Eng. 27, 119-134.

5. Stoll, I., Helm, D., Polanetzki, H. and Wagner, L. (2011) Ultrasonic shot peening (USP) on Ti-6Al-4 V and Ti-6Al-2Sn 4Zr-6Mo aero engine components. ICSP-11.

6. Bhuvaraghan, B., Srinivasan, S., Maffeo, B. and Prakash, O. (2010) Analytical solution for single and multiple impacts with strain-rate effects for shot peening. CMES 57, 137.

7. Yu, C., Gao, H., Yu, H., Jiang, H. and Cheng, G. J. (2009) Laser dynamic forming of functional materials laminated composites on patterned three-dimensional surfaces with applications on flexible microelectromechanical systems. Appl. Phys. Lett. 95, 191108.
8. Ramesh, K. T. (2008) Part D: Chapter 33. High strain rate and impact experiments. Springer Handbook of Experimental Solid Mechanics. Springer - Verlag, New York Inc.

9. Gorham, D. A. (1980) Measurement of stress-strain properties of strong metals at very high strain rates. Inst. Phys. Conf. Ser. 47, 16-24.

10. Othman, R., Guégan, P., Challita, G., LeBreton, D. and Pasco, F. (2009) A modified servo-hydraulic machine for testing at intermediate strain-rates. Int. J. Impact Eng. 36, 460-467.

11. Kolsky, H. (1963) Stress Waves in Solids. Vol. 1098. Courier Dover Publications.

12. Davis, E. D. H. and Hunter, S. C. (1963) The dynamic compression test of solids by the method of the split Hopkinson pressure bar. J. Mech. Phys. Solids 11, 155-179.

13. Mocko, W., Rodriguez-Martinez, J. A., Kowalewski, Z. L. and Rusinek, A. (2012) Compressive viscoplastic response of 6082-T6 and 7075-T6 aluminium alloys under wide range of strain rate at room temperature: experiments and modelling. Strain 48, 498-509.

14. Casem, D. T. (2009) A small diameter Kolsky bar for high-rate compression. In Proc. of the 2009 SEM Annual Conference and Exposition on Experimental and Applied Mechanics, Albuquerque, NM.

15. Nicholas, T. (1981) Tensile testing of materials at high rates of strain. Exp. Mech. 21, 177-185.

16. Duffy, J., Campbell, J. D. and Hawley, R. H. (1971) On the use of a torsional split Hopkinson bar to study rate effects in 1100-0 aluminium. J. Appl. Mech. 38, 83-91.

17. Casem, D. T., Grunschel, S. E. and Schuster, B. E. (2012) Normal and transverse displacement interferometers applied to small diameter Kolsky bars. Exp. Mech. 52, 173-184.

18. Kamler, F., Niessen, P. and Pick, R. J. (1995) Measurement of the behaviour of high-purity copper at very high rates of strain. Xanad. J. Phys. 73, 295-303.

19. Chen, W. and Song, B. (2011) Split Hopkinson (Kolsky) Bar, Design Testing and Applications. Springer - Verlag, New York Inc.

20. Gama, B. A., Lopatnikov, S. L. and Gillespie, J. W. (2004) Hopkinson bar experimental technique: a critical review. Appl. Mech. Rev. 57, 223-250.

21. Cowper, G. and Symonds, P. (1957) Strain hardening and strain-rate effects in the impact loading of cantilever beams. Tech. Rep., Brown University Division of Applied Mathematics.

22. Zerilli, F. and Armstrong, R. (1987) Dislocation-mechanicsbased constitutive relations for material dynamics calculations. J. Appl. Phys. 61, 1816-1825.

23. Johnson, G. R. and Cook, W. H. (1983) A constitutive model and data for metals subjected to large strains, high strain rates and high temperatures. Proceedings of the 7th International Symposium on Ballistics, Vol 21, 541-547.

24. Amarchinta, H. K., Grandhi, R. V., Langer, K. and Stargel, D. S. (2009) Material model validation for laser shock peening process simulation. Modell. Simul. Mater. Sci. Eng. 17, 015010.

25. Lee, W. S. and Lin, C. F. (1998) High-temperature deformation behaviour of Ti6Al4V alloy evaluated by high strain-rate compression tests. J. Mater. Process. Technol. 75, 127-136.

26. Yatnalkar, R. S. (2010) Experimental investigation of plastic deformation of Ti-6Al-4 V under various loading conditions, Master thesis of Ohio State University.

27. (2011) ABAQUS 6.11 Document. CDassault Systemes.

28. Safa, K. and Gary, G. (2010) Displacement correction for punching at a dynamically loaded bar end, IE-1835. Int. J. Impact. Eng. 37, 371-384. 
29. Pochhammer, L. (1876) Uber die fortpflanzungsgeschwindigkeiten kleiner schwingungen in einem unbergrenzten isotropen kreiszylinder. J. für die Reine und Angewande Math. 81, 324-336.

30. Chree, C. (1889) The equations of an isotropic elastic solid in polar and cylindrical co-ordinates their solution and applications. Cambridge Phil. Soc. Trans. 14, 250-369.
31. Othman, R. (2012) Cut-off frequencies induced by the length of strain gauges measuring impact events. Strain 48, 16-20.

32. Malinowski, J. Z., Klepaczko, J. R. and Kowalewski, Z. L. (2007) Miniaturized compression test at very high strain rates by direct impact. Exp. Mech. 47, 451-463.

33. Simo, J. C. and Hughes, T. J. R.. Computational Inelasticity. Springer, New York: 1997. 